\title{
Industrial Application of a Second Order Sliding Mode Observer for Speed and Flux Estimation in Sensorless Induction Motor
}

\author{
Sebastien Solvar, Malek Ghanes, Leonardo Amet, \\ Jean-Pierre Barbot and Gaëtan Santomenna
}

Additional information is available at the end of the chapter

http://dx.doi.org/10.5772/52910

\section{Introduction}

Recently, considerable research efforts are focused on the sensorless Induction Motors (IM) control problem. We refer the reader to [12] for a tutorial account on the topic. Indeed, industries concerned by sensorless $I M$ drives are continuously seeking for cost reductions in their products. The main drawback of $I M$ is the mechanical sensor. The use of such direct speed sensor induces additional electronics, extra wiring, extra space, frequent maintenance, careful mounting and default probability. Moreover, the sensor is vulnerable for electromagnetic noise in hostile environments and has a limited temperature range.

To avoid mechanical sensor (speed, position and load torque) of $I M$, several approaches for the so-called "sensorless control" have attracted a great deal of attention recently (see for example [21], [15], [22], [16], [11], [14], [6], [10], [1], [8], [19]. These methods can be classified into three main strategies.

- Artificial intelligence strategies [22], [19].

- Strategies based on IM spatial saliency methods with fundamental excitation and high frequency signal injection [16], [12].

- Fundamental motor model strategies: adaptive observer [21], Luenberger observe [15], Kalman filter observer [11], high gain observer [14], [6], sliding mode observer [10], [1], interconnected high gain observer [8].

This chapter belongs to the third strategy using mainly observer methods.

First and second strategies have been a subject of growing interest in recent years. For example the second strategy based on IM spatial saliency with extra converters is a robust and physical method. But artificial intelligence and spatial saliency algorithms are quite heavy for basic microprocessors. 
The third strategy that is a powerful observer that can estimate simultaneously variables and parameters of a large class of nonlinear systems doesn't require a very high performance processor for real time implementation but they are often tested at high speed in sensorless $I M$ whereas the main difficulties are mainly at very low frequencies [10], [8].

However for our best of knowledge, examination of the literature on the third strategy shows that the real time computation constraints with a cheapest microprocessors or microprocessors not specially allowed to this task $^{1}$ are not taken into account to deal with industrial applications of sensorless $I M$ including very low frequencies drives.

Meanwhile, compared with other observers, sliding mode technic [20] have attractive advantages of robustness against matching disturbances and, insensitivity to some specific variation of parameters in sliding mode behavior. However, the chattering effect (that is inherent to standard first sliding mode technic) is often an obstacle for practical applications. Higher-Order Sliding Modes (see for example [2], [18] and [5]) are one of the solutions which does not compromise robustness and avoid filtering of estimated variables as considered by other methods.

In this chapter, a second order sliding mode observer for the $I M$ without mechanical sensor is presented for the open problem of sensorless IM drives at very low frequency. This observer converges in finite time and is robust to the variation of parameters. To illustrate the proposed observer, firstly a very simple case is presented in order to exemplified the tuning parameters. Then, to highlight the technological interest of the proposed method and also show the difficulties due to real time computation constraints when a basic microprocessors are used, an industrial application is proposed.

This paper is organized as follows: the section 2 recalls both IM model and unobservability phenomena of IM. In section 3 the super twisting algorithm (second sliding mode observer) is first presented in a simple case and then applied for sensorless IM. After that the section 4 proposes a discrete version of the super twisting observer. In section 5 the experimental results of the proposed observer carried out in an industrial framework are presented. Some conclusions and remarks are drawn in section VII.

\section{Technical background}

\subsection{IM model}

In [4] the following IM model is proposed, in the fixed $(\alpha, \beta)$ frame:

$$
\left\{\begin{array}{l}
\dot{i}_{s \alpha}=-\frac{R_{s} L_{r}^{2}+R_{r} M_{s r}^{2}}{\sigma L_{s} L_{r}^{2}} i_{s \alpha}+\frac{M_{s r}}{\sigma L_{s} L_{r}}\left(\frac{R_{r}}{L_{r}} \phi_{r \alpha}+p \Omega \phi_{r \beta}\right)+\frac{1}{\sigma L_{s}} v_{s \alpha} \\
\dot{i}_{s \beta}=-\frac{R_{s} L_{r}^{2}+R_{r} M_{s r}^{2}}{\sigma L_{s} L_{r}^{2}} i_{s \beta}+\frac{M_{s r}}{\sigma L_{s} L_{r}}\left(\frac{R_{r}}{L_{r}} \phi_{r \beta}-p \Omega \phi_{r \alpha}\right)+\frac{1}{\sigma L_{s}} v_{s \beta} \\
\dot{\phi}_{r \alpha}=\frac{M_{s} r R_{r}}{L_{r}} i_{s \alpha}-\frac{R_{r}}{L_{r}} \phi_{r \alpha}-p \Omega \phi_{r \beta} \\
\dot{\phi}_{r \beta}=\frac{M_{s} r R_{r}}{L_{r}} i_{s \beta}-\frac{R_{r}}{L_{r}} \phi_{r \beta}+p \Omega \phi_{r \alpha} \\
\dot{\Omega}=\frac{p M_{s} r}{J L_{r}}\left(\phi_{r \alpha} i_{s \beta}-\phi_{r \beta} i_{s \alpha}\right)-\frac{f}{J} \Omega-\frac{1}{J} T_{l}
\end{array}\right.
$$

\footnotetext{
${ }^{1}$ The microprocessors may be dedicated to many process tasks as supervision process, communication process in addition to the considered task
} 
As the mechanical position and magnetic variables are unknown, $d-q$ frame is well appropriate for sensorless observer based control design.

\section{IM parameters:}

- $R_{S}$ : Stator resistance (Ohms).

- $R_{R}$ : Rotor resistance (Ohms).

- $L_{S}$ : Stator inductance (Ohms).

- $L_{R}$ : Rotor inductance $(H)$.

- $L_{M}$ : Mutual inductance $(H)$.

- $p$ : number of pole pairs.

- $f$ : viscous friction coefficient (Nm.s/ rad).

- $J$ : inertia $\left(K g \cdot \mathrm{m}^{2}\right)$.

\section{IM variables :}

- $v_{s \alpha, \beta}$ : Stator voltage $(\mathrm{V})$.

- $i_{s \alpha, \beta}$ : Stator current (A).

- $\phi_{r \alpha, \beta}$ : Rotor flux $(\mathrm{Wb})$.

- $\Omega$ : Mechanical speed ( $\mathrm{rad} / \mathrm{s})$.

- $T_{l}$ : Load torque (Nm).

In order to construct the proposed observer for an industrial application, we work with a per unit model, under the following equations :

$$
\left\{\begin{array}{l}
\dot{x}_{1}=-\gamma x_{1}+\theta\left(b x_{3}+c x_{5} x_{4}\right)+\xi v_{1} \\
\dot{x}_{2}=-\gamma x_{2}+\theta\left(b x_{4}-c x_{5} x_{3}\right)+\xi v_{2} \\
\dot{x}_{3}=a x_{1}-b x_{3}-c x_{5} x_{4} \\
\dot{x}_{4}=a x_{2}-b x_{4}+c x_{5} x_{3} \\
\dot{x}_{5}=h\left(x_{3} x_{2}-x_{4} x_{1}\right)-d x_{5}-e T_{l}
\end{array}\right.
$$

With the following parameters:

$$
\begin{aligned}
& x_{1}=\frac{i_{s \alpha}}{I_{r e f}} \\
& x_{2}=\frac{i_{s \beta}}{I_{r e f}} \\
& a=\frac{M_{s r} I_{r e f} \omega_{r e f}}{T_{r} V_{r e f}} \quad b=\frac{1}{\tau_{r}} \\
& x_{3}=\frac{\omega_{r e f} \phi_{r \alpha}}{V_{r e f}} \\
& x_{4}=\frac{\omega_{r e f} \phi_{r \beta}}{V_{r e f}} \quad x_{5}=\frac{p \Omega}{\omega_{r e f}} \\
& e=\frac{p}{J \omega_{r e f}} \\
& c=\omega_{r e f} \\
& d=\frac{f_{v}}{J} \\
& h=\frac{p^{2} M_{s r} I_{r e f} V_{r e f}}{J \omega_{r e f}^{2} L_{r}} \\
& \theta=\frac{K V_{\text {ref }}}{I_{\text {ref }} \omega_{\text {ref }}} \\
& \xi=\frac{V_{r e f}}{\sigma L_{s} I_{r e f}} \\
& \sigma=1-\frac{M_{s r}^{2}}{L_{s} L_{r}} \\
& \gamma=\frac{R_{s} L_{r}^{2}+R_{r} M_{s r}^{2}}{\sigma L_{s} L_{r}^{2}} \\
& \tau_{r}=\frac{L_{r}}{R_{r}} \\
& K=\frac{M_{s r}}{\sigma L_{s} L_{r}}
\end{aligned}
$$

Thus for the sake of homogeneity, hereafter experimental results will be given in per-unit (p.u.). 


\subsection{Observability}

The IM observability has been studied by several authors (see for example [3], [13], [9]). In [9], it is proved that the IM observability cannot be established in the particular case when fluxes $\Phi_{r \alpha}, \Phi_{r \beta}$ and speed $\Omega$ are constant, even if we use the higher derivatives of currents. This is a sufficient and necessary condition for lost of observability.

This operating case match to the following physically interpretation:

Constant fluxes $\left(\dot{\phi}_{r \alpha}=\dot{\phi}_{r \beta}=0\right)$

With $\omega_{s}$ the stator voltage pulsation and $T_{e m}$ the electromagnetic torque.

$$
\omega_{s}=p \Omega+\frac{R_{r} T_{e m}}{p \Phi_{r d}^{2}}=0
$$

where $\Phi_{r d}^{2}=\phi_{r \alpha}^{2}+\phi_{r \beta}^{2}$ is the square of the direct flux in $(\mathrm{d}, \mathrm{q})$ frame.

Constant speed $(\dot{\Omega}=0)$

$$
T_{e m}=f \Omega+T_{l}
$$

Thanks to previous equations, we obtain:

$$
T_{l}=-\left(f+\frac{p^{2} \Phi_{r d}^{2}}{R_{r}}\right) \Omega
$$

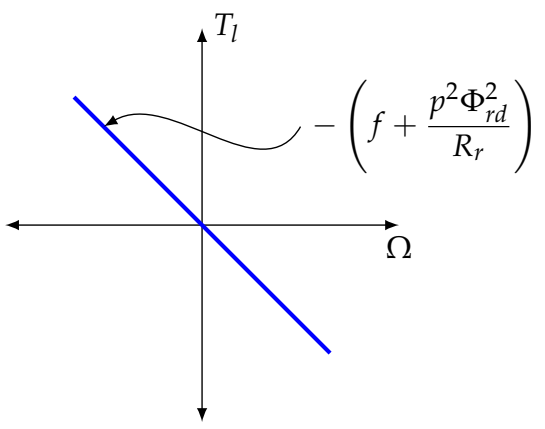

Figure 1. Inobservability curve

The unobservability curve in the map $\left(T_{l}, \Omega\right)$ is shown in figure (1).

Obviously, the observability is lost gradually when we approach this curve [9].

\section{Second order sliding mode observer}

\subsection{Super twisting algorithm: An academic example}

Sliding modes were used at first, as a control technique, but in the recent years it presented as a very good tool for observer design [17], [17], [5]. 
Considering the following system:

$$
\left\{\begin{array}{l}
\dot{x}_{1}=x_{2} \\
\dot{x}_{2}=f(x, t) \\
y=h(x)=x_{1}
\end{array}\right.
$$

With $f(x, t)$ a bounded function.

For system (6), a second order sliding mode observer is designed in the following way:

$$
\left\{\begin{array}{l}
\dot{\hat{x}}_{1}=\hat{x}_{2}+\lambda\left|e_{1}\right|^{\frac{1}{2}} \operatorname{sign}\left(e_{1}\right) \\
\dot{\hat{x}}_{2}=\alpha \operatorname{sign}\left(e_{1}\right)
\end{array}\right.
$$

$$
\text { With } \lambda, \alpha>0 \quad \text { and } \quad e_{1}=x_{1}-\hat{x}_{1} \text {. }
$$

The efficiency of the this strategy depends on coefficients $\alpha$ and $\lambda$. For second order system (6)we show convergence of estimated variables $\left(\hat{x}_{1}, \hat{x}_{2}\right)$ to $\left(x_{1}, x_{2}\right)$ by studying dynamics errors $\dot{e}_{1}$ and $\dot{e}_{2}$.

Thus

$$
\left\{\begin{array}{l}
\dot{e}_{1}=\dot{x}_{1}-\dot{\hat{x}}_{1}=e_{2}-\lambda\left|x_{1}-\hat{x}_{1}\right|^{\frac{1}{2}} \operatorname{sign}\left(x_{1}-\hat{x}_{1}\right) \\
\dot{e}_{2}=\dot{x}_{2}-\dot{\hat{x}}_{2}=f(x, t)-\alpha \operatorname{sign}\left(x_{1}-\hat{x}_{1}\right)
\end{array}\right.
$$

With

$$
f(x, t) \in\left[-f^{+}, f^{+}\right], \quad e_{2}=x_{2}-\hat{x}_{2}
$$

And

$$
\ddot{e}_{1}=f(x, t)-\alpha \operatorname{sign}\left(e_{1}\right)-\frac{1}{2} \lambda\left|e_{1}\right|^{-\frac{1}{2}} \dot{e}_{1}
$$

Thus

$$
\ddot{e}_{1} \in\left[-f^{+}, f^{+}\right]-\alpha \operatorname{sign}\left(e_{1}\right)-\frac{1}{2} \lambda\left|e_{1}\right|^{-\frac{1}{2}} \dot{e}_{1}
$$

Where

$$
f^{+}=\max \{f(x, t)\}
$$

Conditions on $\lambda$ and $\alpha$ that permit a convergence in finite time of $\left(\dot{e}_{1}, e_{1}\right)$ to $(0,0)$ are derived hereafter according to figure 2 .

Proposition: For any initial conditions $x(0), \hat{x}(0)$, there exists a choice of $\lambda$ and $\alpha$ such that the error dynamics $\dot{e}_{1}$ and $\dot{e}_{2}$ converge to zero in finite time and by consequence $\hat{x}_{1} \longmapsto x_{1}$ and $\tilde{x}_{2} \longmapsto x_{2}$. 


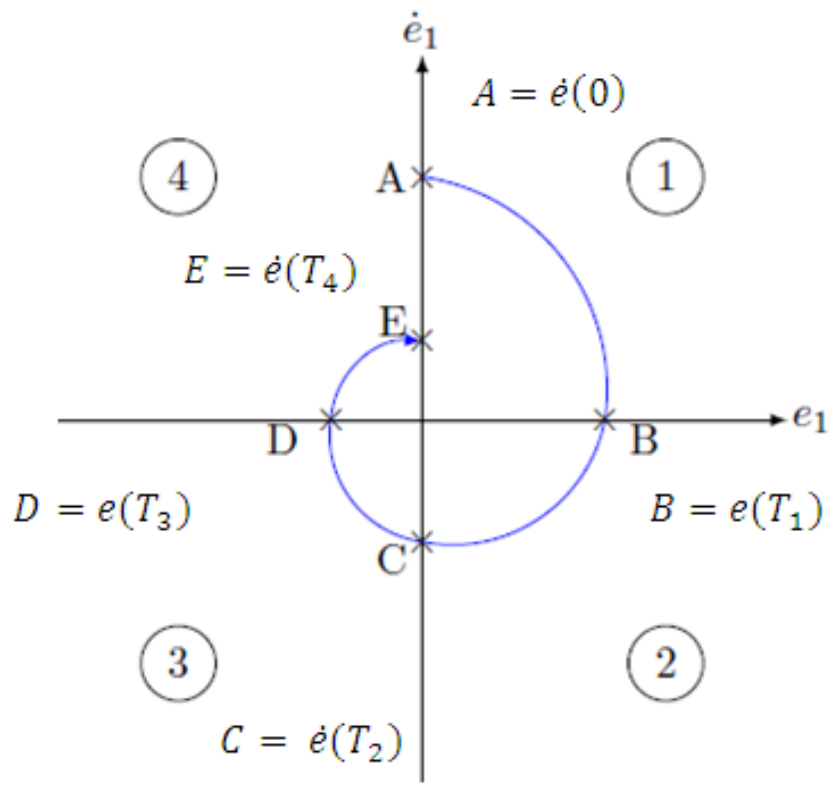

Figure 2. Upper bound of finite time convergence curve.

Proof: Consider system (6). To show the convergence of $\left(\hat{x}_{1}, \hat{x}_{2}\right)$ to $\left(x_{1}, x_{2}\right)\left(\right.$ ie., $\left(e_{1}, e_{2}\right) \rightarrow$ $(0,0))$, we need to show that

$$
\frac{\left|\dot{e}_{1}\left(T_{2}\right)\right|}{\left|\dot{e}_{1}(0)\right|}<1
$$

of figure 2 , where $\dot{e}_{1}\left(T_{2}\right)=C$ and $\dot{e}_{1}(0)=A$.

Figure 2 illustrates the finite time convergence behavior of the proposed observer for system 6. In what follows we will give the error trajectory for each quadrant in the worst cases.

Let consider the system's dynamic $\ddot{e}_{1}$

$$
\ddot{e}_{1}=f(x, t)-\alpha \operatorname{sign}\left(e_{1}\right)-\frac{\lambda}{2}\left|e_{1}\right|^{-\frac{1}{2}} \dot{e}_{1}
$$

with $\frac{d|x|}{d t}=\dot{x} \operatorname{sign}(x)$.

Equation (10) leads to

$$
\ddot{e}_{1} \in\left[-f^{+}, f^{+}\right]-\alpha \operatorname{sign}\left(e_{1}\right)-\frac{\lambda}{2}\left|e_{1}\right|^{-\frac{1}{2}} \dot{e}_{1}
$$

where

$$
f^{+}=\max (f(t, x)),
$$


First quadrant: $e_{1}>0$ and $\dot{e}_{1}>0$

Starting from point A of figure 2 the trajectory of $\dot{e}_{1}=f\left(e_{1}\right)$ is in the first quadrant $e_{1} \geq 0$ and $\dot{e}_{1} \geq 0$. The rising trajectory is given by $\ddot{e}_{1}=-\left(\alpha-f^{+}\right)$.

By choosing

$$
\alpha>f^{+}
$$

we ensure that $\ddot{e}_{1}<0$ and hence $\dot{e}_{1}$ decreases and tends towards the y-axis, corresponding to $\dot{e}_{1}=0$ (point $\mathrm{B}$ in figure 2).

\section{Computing of $e_{1}\left(T_{1}\right)$}

From (10), we have

$$
\ddot{e}_{1}=-\left(\alpha-f^{+}\right)
$$

Which implies that

$$
\dot{e}_{1}(t)=-\left(\alpha-f^{+}\right) t+\dot{e}_{1}(0)
$$

And

$$
e_{1}(t)=-\left(\alpha-f^{+}\right) \frac{t^{2}}{2}+\dot{e}_{1}(0) t
$$

From (13), since $\dot{e}_{1}\left(T_{1}\right)=0$, we obtain the necessary time for going from $A$ to $B$ with $B=$ $e_{1}\left(T_{1}\right)$.

$$
T_{1}=\frac{\dot{e}_{1}(0)}{\left(\alpha-f^{+}\right)}
$$

Then we can compute $e\left(T_{1}\right)$ as follows

$$
\begin{aligned}
e_{1}\left(T_{1}\right) & =-\left(\alpha-f^{+}\right) \frac{\dot{e}_{1}^{2}(0)}{2\left(\alpha-f^{+}\right)}+\frac{\ddot{e}_{1}^{2}(0)}{\left(\alpha-f^{+}\right)} \\
& =\frac{\dot{e}_{1}^{2}(0)}{2\left(\alpha-f^{+}\right)}
\end{aligned}
$$

Second quadrant: $e_{1}>0$ and $\dot{e}_{1}<0$

In this case, $\ddot{e}_{1}=-f^{+}-\alpha \operatorname{sign}\left(e_{1}\right)-\frac{\lambda}{2}\left|e_{1}\right|^{-\frac{1}{2}} \dot{e}_{1}$

becomes negative $\left(\ddot{e}_{1}<0\right)$ on making a good choice of $\alpha$ which leads to

$$
\left(\alpha+f_{1}^{+}\right)>-\frac{\lambda_{1}}{2}\left|e_{1}\right|^{-\frac{1}{2}} \dot{e}_{1}
$$

Since $\dot{e}_{1}$ is negative, then

$$
\left|\dot{e}_{1}(t)\right| \leq \frac{2\left(\alpha+f_{1}^{+}\right)}{\lambda}\left|e_{1}(t)\right|^{\frac{1}{2}}
$$


Considering by the sake of simplicity (17), $e_{1}>0$ and $\dot{e}_{1}<0$.

Integrating (17) with $e_{1}(0)=0$ gives

$$
\sqrt{e_{1}(t)}=\frac{\left(\alpha+f^{+}\right)}{\lambda} t
$$

At $t=T_{2}$, we should make the inverse of function (18) from point $B$ to $C$ in figure 2. This leads to

$$
e_{1}\left(T_{2}\right)=e_{1}\left(T_{1}\right)
$$

Then (18) becomes

$$
\sqrt{e_{1}\left(T_{1}\right)}=\frac{\left(\alpha+f^{+}\right)}{\lambda} T_{2}
$$

By replacing $e_{1}\left(T_{1}\right)$ coming from (15) in equation (20), we get the necessary time for going from $B$ to $C$

$$
T_{2}=\frac{\lambda}{\left(\alpha+f^{+}\right)} \frac{\dot{e}_{1}(0)}{\sqrt{2}\left(\alpha-f^{+}\right)}
$$

After that, by using the argument of (19) in equation (17) evaluated at $t=T_{2}$ in the worth case, we get

$$
\left|\dot{e}_{1}\left(T_{2}\right)\right|=\frac{2\left(\alpha+f_{1}^{+}\right)}{\lambda}\left|e_{1}\left(T_{1}\right)\right|^{\frac{1}{2}}
$$

By replacing $e_{1}\left(T_{1}\right)$ by its expression given by (15) in (22), we get

$$
\left|\dot{e}_{1}\left(T_{2}\right)\right|=\frac{2\left(\alpha+f_{1}^{+}\right)}{\lambda} \frac{\left|\dot{e}_{1}(0)\right|}{\sqrt{2} \sqrt{\left(\alpha-f^{+}\right)}}
$$

Thus, by satisfying inequality (9) in equation (23) $\lambda$ should be chosen as

$$
\lambda>\left(\alpha+f_{1}^{+}\right) \sqrt{\frac{2}{\left(\alpha-f^{+}\right)}}
$$

Finally, conditions (12) and (24) of the observer parameters are sufficient conditions guaranteeing the state convergence (i.e. the states $\left(e_{1}, \dot{e}_{1}\right)$ tend towards $e_{1}=\dot{e}_{1}=0$ (Figure 2).

This ends the proof.

Moreover the convergence is in finite time, because from (14) and 21 we obtain

$$
T_{\infty} \leq\left(\sum_{i=0}^{+\infty}\left(\frac{\sqrt{2}\left(\alpha+f^{+}\right)}{\lambda \sqrt{\alpha-f^{+}}}\right)^{i}\right)\left(\frac{\lambda}{\sqrt{2}\left(\alpha+f^{+}\right)}+1\right) \frac{\dot{e}_{1}(0)}{\left(\alpha-f^{+}\right)}
$$

as

$$
\left|\frac{\sqrt{2}\left(\alpha+f^{+}\right)}{\lambda \sqrt{\alpha-f^{+}}}\right|<1
$$


we obtain a bounded limit

$$
T_{\infty} \leq\left(\frac{1}{1-\frac{\sqrt{2}\left(\alpha+f^{+}\right)}{\lambda \sqrt{\alpha-f^{+}}}}\right)\left(\frac{\lambda}{\sqrt{2}\left(\alpha+f^{+}\right)}+1\right) \frac{\dot{e}_{1}(0)}{\left(\alpha-f^{+}\right)}
$$

Here we give simulations of a very simple example. The function $f(t, x)$ in system (6) is set equal to $\sin (t)$ with $f^{+}=\max \{\sin (t)\}=1$. We get

$$
\left\{\begin{array}{l}
\dot{x}_{1}=x_{2} \\
\dot{x}_{2}=\sin (t) \\
y=x_{1}
\end{array}\right.
$$

The associated observer is:

$$
\left\{\begin{array}{l}
\dot{\hat{x}}_{1}=\tilde{x}_{2}+\lambda\left|e_{1}\right|^{\frac{1}{2}} \operatorname{sign}\left(e_{1}\right) \\
\dot{\tilde{x}}_{2}=\alpha \operatorname{sign}\left(e_{1}\right)
\end{array}\right.
$$

The simulation results are shown in figure 3. It can be seen that figure (3) spotlight two steps into Super Twisting Algorithm, which are convergence step in finite time, and sliding mode. Indeed observer is working on $t=1 \mathrm{~s}$ with $\hat{x}_{1}(0)=1$ and $\tilde{x}_{2}(0)=1$. $\hat{x}_{1}$ converges under $1 s$ to $x_{1}$, and then slides along $x_{1}$ path, and equal to $\tilde{x}_{2}$.

\subsection{Application to Induction Motor}

At first, due to the nonlinearity of flux and speed product, the IM model (2) is not written in a suitable form allowing to apply the super twisting algorithm presented in previous section. To overcome this difficulty, we make the following change of variables in order to rewrite the IM model (2) (without $\dot{x}_{5}$ equation) into a form 6:

$$
\left\{\begin{array}{l}
z_{1}=x_{1} \\
z_{2}=x_{2} \\
z_{3}=b x_{3}+c x_{5} x_{4} \\
z_{4}=b x_{4}-c x_{5} x_{3} \\
z_{5}=\dot{z}_{3} \\
z_{6}=\dot{z}_{4}
\end{array}\right.
$$

Equation 27 is not a diffeomorphism, not an homeomorphism but only an immersion, because the dimension of $x$ is 5 and the dimension of $z$ is 6 . Nevertheless, this immersion is used in order to avoid some singularities in a speed estimation as this will be pointed out in the next.

From the IM model (2) and (27), we obtain a new dynamical system as following:

$$
\left\{\begin{array}{l}
\dot{z}_{1}=-z_{1}+\theta z_{3}+\xi v_{1} \\
\dot{z}_{2}=-\gamma z_{2}+\theta z_{4}+\xi v_{2} \\
\dot{z}_{3}=z_{5} \\
\dot{z}_{4}=z_{6}
\end{array}\right.
$$



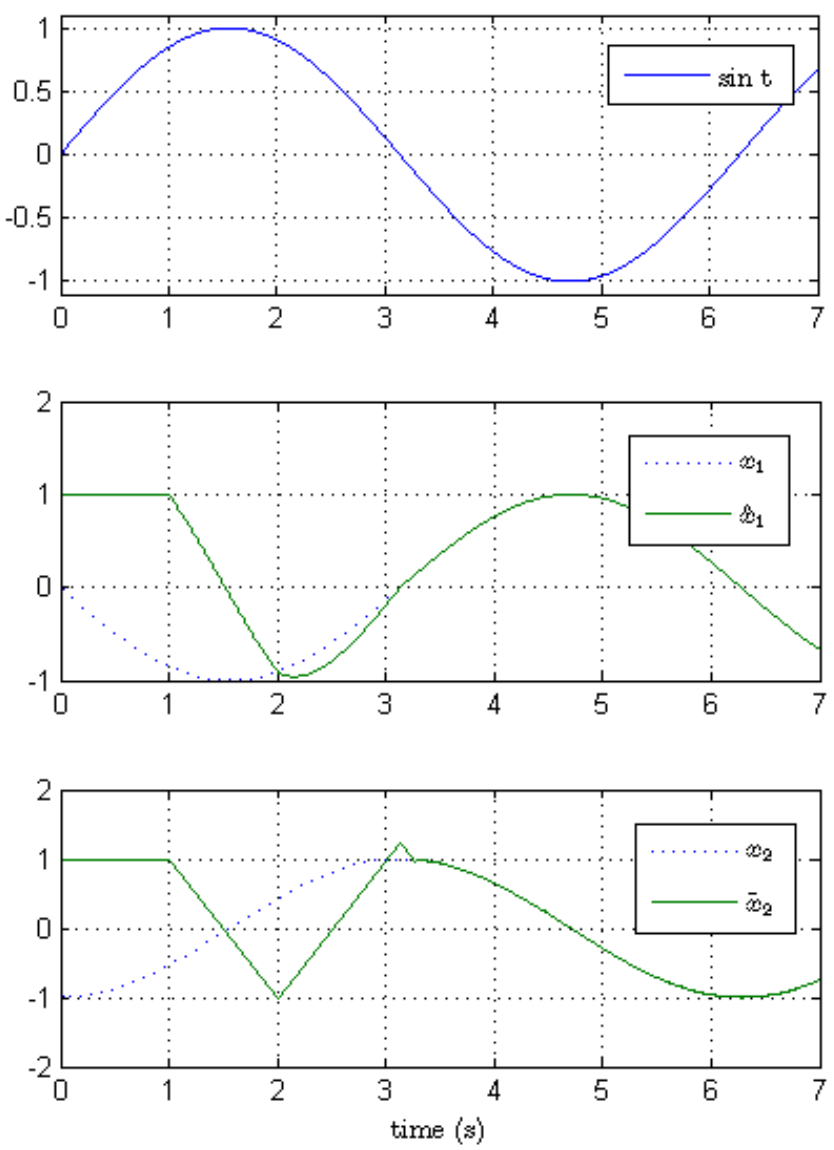

Figure 3. Super Twisting Algorithm example.

Thus, we can propose a new observer structure for dynamical system (28):

$$
\begin{aligned}
& \left\{\begin{array}{l}
\dot{\hat{z}}_{1}=\theta \tilde{z}_{3}-\gamma z_{1}+\xi v_{1}+\lambda_{1}\left|e_{1}\right|^{\frac{1}{2}} \operatorname{sign}\left(e_{1}\right) \\
\dot{\tilde{z}}_{3}=\alpha_{1} \operatorname{sign}\left(e_{1}\right) \\
\dot{\hat{z}}_{2}=\theta \tilde{z}_{4}-\gamma z_{2}+\xi v_{2}+\lambda_{2}\left|e_{2}\right|^{\frac{1}{2}} \operatorname{sign}\left(e_{2}\right) \\
\dot{\tilde{z}}_{4}=\alpha_{2} \operatorname{sign}\left(e_{2}\right) \\
\dot{\hat{z}}_{3}=E_{1} E_{2}\left(\tilde{z}_{5}+\lambda_{3}\left|e_{3}\right|^{\frac{1}{2}} \operatorname{sign}\left(e_{3}\right)\right) \\
\dot{\tilde{z}}_{5}=E_{1} E_{2} \alpha_{3} \operatorname{sign}\left(e_{3}\right) \\
\dot{\hat{z}}_{4}=E_{1} E_{2}\left(\tilde{z}_{6}+\lambda_{4}\left|e_{4}\right|^{\frac{1}{2}} \operatorname{sign}\left(e_{4}\right)\right) \\
\dot{\tilde{z}}_{6}=E_{1} E_{2} \alpha_{4} \operatorname{sign}\left(e_{4}\right)
\end{array}\right. \\
& \text { with } E_{i}\left\{\begin{array}{l}
1 \text { if } e_{i}=z_{i}-\hat{z}_{i}=0, i=1,2 \\
0 \text { if not }
\end{array}\right.
\end{aligned}
$$


This observer structure depends on Super Twisting Algorithm presented in previous section and Step by Step proficiencies [7]. We propose to put in multiples-series observers with functions $\left(E_{i}\right)$.
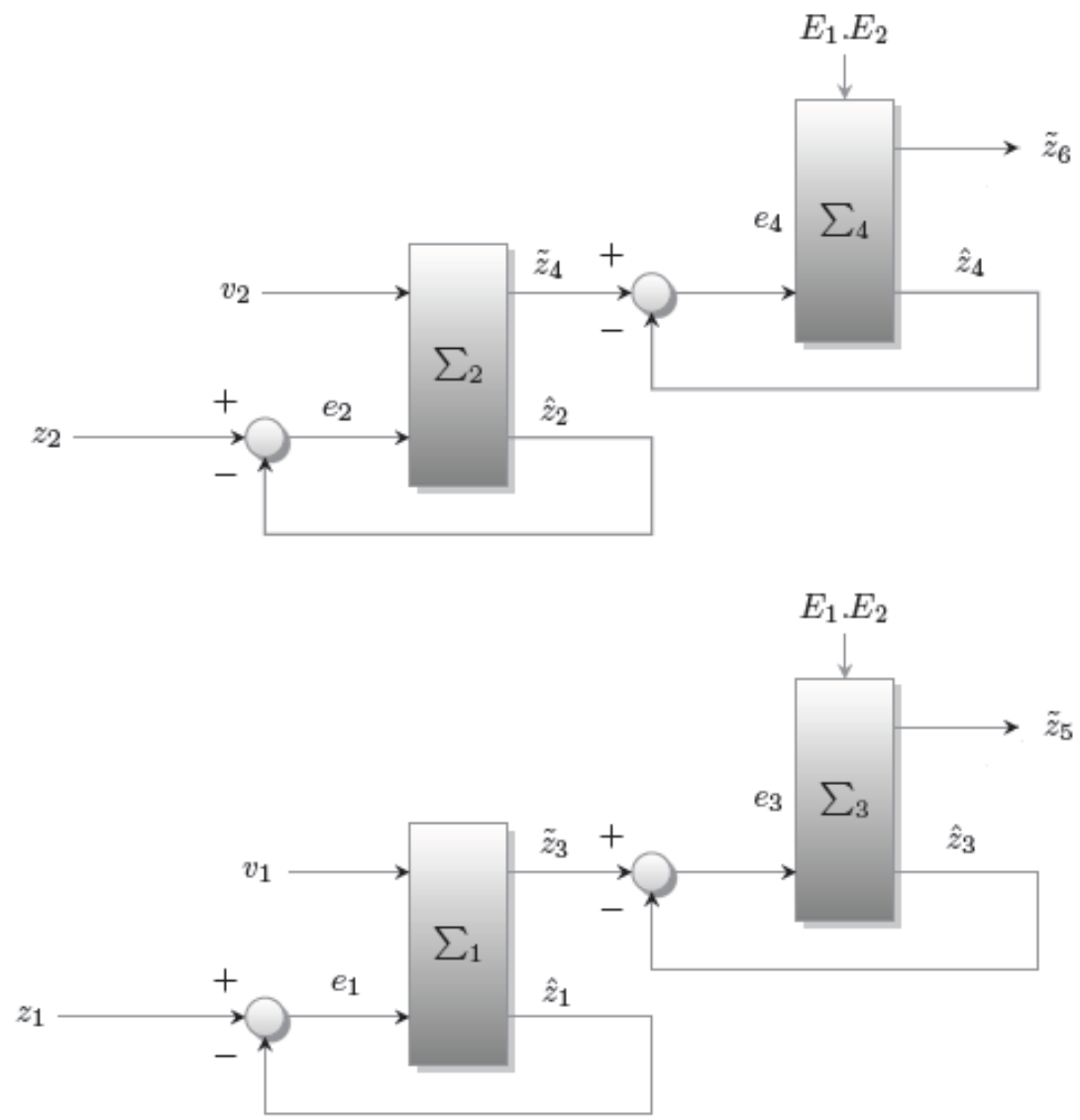

Figure 4. General IM Observer Structure.

The functions $E_{i}$ ensure that the next steps errors do not escape too far before one has the convergent of the last step error.

The gains $\alpha_{i}, \lambda_{i}$ are chosen which respect to the reachability condition of the Super Twisting algorithm as stated in inequalities (12) and (24) of previous section. By choosing

$$
\begin{aligned}
& \alpha_{1}>\max \left(\theta z_{3}\right), \quad \lambda_{1}>\left(\max \left(\theta z_{3}\right)+\alpha_{1}\right) \sqrt{\frac{2}{\alpha_{1}-\max \left(\theta z_{3}\right)}} \\
& \alpha_{2}>\max \left(\theta z_{4}\right), \quad \lambda_{2}>\left(\max \left(\theta z_{4}\right)+\alpha_{2}\right) \sqrt{\frac{2}{\alpha_{2}-\max \left(\theta z_{4}\right)}}
\end{aligned}
$$




$$
\begin{aligned}
& \alpha_{3}>\max \left(z_{5}\right), \quad \lambda_{3}>\left(\max \left(z_{5}\right)+\alpha_{3}\right) \sqrt{\frac{2}{\alpha_{3}-\max \left(z_{5}\right)}} \\
& \alpha_{4}>\max \left(z_{6}\right), \quad \lambda_{4}>\left(\max \left(z_{6}\right)+\alpha_{4}\right) \sqrt{\frac{2}{\alpha_{4}-\max \left(z_{6}\right)}}
\end{aligned}
$$

and we get

$$
e_{1}=e_{2}=e 3=e_{4}=0
$$

i.e.

$$
\begin{array}{ll}
\hat{z}_{1}=z_{1}, & \hat{z}_{2}=z_{2} \\
\hat{z}_{3}=\tilde{z}_{3}, & \hat{z}_{4}=\tilde{z}_{4} \\
\tilde{z}_{5}=z_{5}, & \tilde{z}_{6}=z_{6}
\end{array}
$$

Consequently all variables $z_{1}, z_{2}, \hat{z}_{3}, \hat{z}_{4}, \tilde{z}_{5}, \tilde{z}_{6}$ are available and then we can deduce IM variables.

We propose to treat this problem in two different cases: $\dot{x}_{5} \neq 0$, and $\dot{x}_{5}=0$

CASE A : $\dot{x}_{5} \neq 0$

Firstly we propose to express fluxes $x_{3}$ and $x_{4}$, from equation (27) we obtain:

$$
\begin{aligned}
& \hat{z}_{3}=b x_{3}+c x_{5} x_{4} \\
& \hat{z}_{4}=b x_{4}-c x_{5} x_{3}
\end{aligned}
$$

We deduce

$$
\begin{aligned}
& x_{3}=\frac{\hat{z}_{3}-c x_{5} x_{4}}{b} \\
& x_{4}=\frac{\hat{z}_{4}+c x_{5} x_{3}}{b}
\end{aligned}
$$

By substituting $x_{4}$ by its expression in (35) and $x_{3}$ in (36) we have:

$$
\begin{aligned}
& x_{3}=\frac{\hat{z}_{4}+\frac{c}{b} z_{3} x_{5}}{b+\frac{c^{2} x_{5}^{2}}{b}} \\
& x_{4}=\frac{\hat{z}_{3}-\frac{c}{b} z_{4} x_{5}}{b+\frac{c^{2} x_{5}^{2}}{b}}
\end{aligned}
$$

Now let us express $x_{5}$. From (2) we know

$$
\begin{aligned}
& \dot{x}_{3}=a x_{1}-b x_{3}-c x_{5} x_{4} \\
& \dot{x}_{4}=a x_{2}-b x_{4}+c x_{5} x_{3}
\end{aligned}
$$


Firstly we propose to write $\dot{x}_{3}$ and $\dot{x}_{4}$ as a function of variables $z$. By using (27) in (38), we get:

$$
\begin{aligned}
& \dot{x}_{3}=a z_{1}-\hat{z}_{3} \\
& \dot{x}_{4}=a z_{2}-\hat{z}_{4}
\end{aligned}
$$

By Replacing (39) in (38) and using the two first equations in (27), it follows

$$
\begin{aligned}
& \hat{z}_{3}=b x_{3}+c x_{5} x_{4} \\
& \hat{z}_{4}=b x_{4}-c x_{5} x_{3}
\end{aligned}
$$

Taking the time derivative of (41) and using third-fourth equations in (27) yields to

$$
\begin{aligned}
& \dot{z}_{3}=\tilde{z}_{5}=b \dot{x}_{3}+c \dot{x}_{5} x_{4}+c \dot{x}_{4} x_{5} \\
& \dot{z}_{4}=\tilde{z}_{6}=b \dot{x}_{4}-c \dot{x}_{5} x_{3}-c \dot{x}_{3} x_{5}
\end{aligned}
$$

From (42) we have:

$$
\dot{x}_{5}=\frac{\tilde{z}_{5}-b \dot{x}_{3}-c x_{5} \dot{x}_{4}}{c x_{4}}
$$

By substituting (44) in (43), we get

$$
\tilde{z}_{6}=b \dot{x}_{4}-c x_{5} \dot{x}_{3}-x_{3} \frac{\tilde{z}_{5}-b \dot{x}_{3}-c x_{5} \dot{x}_{4}}{x_{4}}
$$

Then we can deduce the motor speed $x_{5}$ by replacing in (45) expressions of $x_{3}-x_{4}$ and $\dot{x}_{3}-\dot{x}_{4}$ coming from (37) and (39), respectively.

After a straightforward computations, we obtain a second order expression of $x_{5}$ :

$$
\pi_{1} x_{5}^{2}+\pi_{2} x_{5}+\pi_{3}=0
$$

where

$$
\begin{aligned}
& \pi_{1}=\frac{c}{b}\left[\left(a z_{2}-\hat{z}_{4}\right) \hat{z}_{3}-\left(a z_{1}-\hat{z}_{3}\right) \hat{z}_{4}\right] \\
& \pi_{2}=\frac{c}{b}\left[b\left(a z_{1}-\hat{z}_{3}\right) \hat{z}_{3}-b\left(a z_{2}-\hat{z}_{4}\right) \hat{z}_{4}-\tilde{z}_{5} \hat{z}_{3}+\hat{z}_{4} \tilde{z}_{6}\right] \\
& \pi_{3}=\hat{z}_{3}\left[-\tilde{z}_{6}+b\left(a z_{2}-\hat{z}_{4}\right)\right]-\hat{z}_{4}\left[\tilde{z}_{5}-b\left(a z_{1}-\hat{z}_{3}\right)\right]
\end{aligned}
$$

CASE B : $\dot{x}_{5}=0$

We propose this hypothesis because of dynamical gap evolution between electrical and mechanical variables, in fact speed evolves much more slowly than currents or fluxes.

Thus with this hypothesis we simplify (42) and (43), and obtain two expressions of $x_{5}$ :

$$
x_{5}=\frac{\tilde{z}_{5}-b \dot{x}_{3}}{c \dot{x}_{4}}
$$

or

$$
x_{5}=\frac{b \dot{x}_{4}-\tilde{z}_{6}}{c \dot{x}_{3}}
$$


we change $\dot{x}_{3}$ by expression (35) and $\dot{x}_{4}$ by expression(36)

$$
x_{5}=\frac{\tilde{z}_{5}-b a z_{1}+b \hat{z}_{3}}{c a z_{2}-c \hat{z}_{4}}
$$

or

$$
x_{5}=\frac{\tilde{z}_{6}+b a z_{2}-b \hat{z}_{4}}{c a z_{1}-c \hat{z}_{3}}
$$

Equations (49) and (50) are true only if :

$$
c a z_{2}-c \hat{z}_{4} \neq 0 \text { for (49) and } c a z_{1}-c \hat{z}_{3} \neq 0 \text { for (50) }
$$

\section{Speed estimation}

In order to avoid singularities of speed estimation in (49) and (50), we use the fact that (49) and (50) are in quadrature and thus we get the estimation of speed $x_{5}$ as follows :

$$
x_{5}=\frac{\left(\tilde{z}_{5}-b a z_{1}+b \hat{z}_{3}\right)\left(c a z_{2}-c \hat{z}_{4}\right)+\left(\tilde{z}_{6}+b a z_{2}-b \hat{z}_{4}\right)\left(c a z_{1}-c \hat{z}_{3}\right)}{\left(c a z_{2}-c \hat{z}_{4}\right)^{2}+\left(c a z_{1}-c \hat{z}_{3}\right)^{2}}
$$

\section{Flux estimation}

The rotor flux are obtained by replacing the estimation speed (51) in (37)

\section{Flux position estimation}

Having the rotor flux estimation (37), we can obtain rotor flux position $\rho$

$$
\rho=\operatorname{atan}\left(\frac{x_{4}}{x_{3}}\right)
$$

\section{Discrete time implementation}

\subsection{Explicit Euler method}

For the industrial application in real time, the discrete time observer is designed. The explicit Euler's method is chosen to transform continuous observer to discrete observer. This is due to the simplicity of computation. Considering a differential equation :

$$
\dot{x}=f(x)
$$

The explicit Euler's method with a sampling time $T_{e}$ gives:

$$
x(k)=x(k-1)+T_{e} f(x(k-1))
$$

the data acquisition period $T_{e}$ is also the computation period.

Applying the explicit Euler's method for the second order sliding mode observer, the discrete observer is obtained: 


$$
\left\{\begin{aligned}
& \hat{z}_{1}(k)=\hat{z}_{1}(k-1)+T_{e}\left[\theta \tilde{z}_{3}(k-1)-\gamma z_{1}(k-1)+\xi v_{1}(k-1)\right. \\
&\left.+\lambda_{1}\left|e_{1}(k-1)\right|^{\frac{1}{2}} \operatorname{sign}\left(e_{1}(k-1)\right)\right] \\
& \tilde{z}_{3}(k)=\tilde{z}_{3}(k-1)+T_{e} \alpha_{1} \operatorname{sign}\left(e_{1}(k-1)\right) \\
& \hat{z}_{2}(k)=\hat{z}_{2}(k-1)+T_{e}\left[\theta \tilde{z}_{4}(k-1)-\gamma z_{2}(k-1)+\xi v_{2}(k-1)\right. \\
&\left.+\lambda_{2}\left|e_{2}(k-1)\right|^{\frac{1}{2}} \operatorname{sign}\left(e_{2}(k-1)\right)\right] \\
& \tilde{z}_{4}(k)=\tilde{z}_{4}(k-1)+T_{e} \alpha_{2} \operatorname{sign}\left(e_{2}(k-1)\right) \\
& \hat{z}_{3}(k)=\tilde{z}_{3}(k-1)+T_{e} E_{1} E_{2}\left[\tilde{z}_{5}(k-1)+\lambda_{3}\left|e_{3}(k-1)\right|^{\frac{1}{2}} \operatorname{sign}\left(e_{3}(k-1)\right)\right] \\
& \tilde{z}_{5}(k)=\tilde{z}_{5}(k-1)+T_{e} E_{1} E_{2} \alpha_{3} \operatorname{sign}\left(e_{3}(k-1)\right) \\
& \hat{z}_{4}(k)=\hat{z}_{4}(k-1)+T_{e} E_{1} E_{2}\left[\tilde{z}_{6}(k-1)+\lambda_{4}\left|e_{4}(k-1)\right|^{\frac{1}{2}} \operatorname{sign}\left(e_{4}(k-1)\right)\right] \\
& \tilde{z}_{6}(k)=\tilde{z}_{6}(k-1)+T_{e} E_{1} E_{2} \alpha_{4} \operatorname{sign}\left(e_{4}(k-1)\right)
\end{aligned}\right.
$$

\subsection{Oversampling}

To achieve good accuracy, a small sample period and fast DSP are needed. In the industrial application, the DSP clock frequency is only $150 \mathrm{MHz}$, which does not allow a small enough sample period. So in experimentation an over-sample technique is proposed. In the following paragraphs we show that, under a few low restrictive conditions, it is possible to reduce the error of Euler's method, seen in the previous subsection (4.1).

Hereafter we first present the oversampling method in a very simple use, where $f \in C^{\infty}$. Assume a continuous autonomous system of the form:

$$
\dot{x}=f(x) ; \quad x\left(t_{0}\right)=x_{0}
$$

Assume in addition that the system is discretized at a sampling time $T_{e}$. Then the system ((54)) can be approximated by the explicit Euler's method:

$$
x\left(t_{k+1}\right)=x\left(t_{k}\right)+T_{e} \dot{x}\left(t_{k}\right)+O\left(T_{e}^{2}\right)
$$

For small values of $T_{e}, O\left(T_{e}^{3}\right)$ is neglected and the truncation error is approximately proportional to $T_{e}^{2}$.

Suppose now that the system (54) is discretized at two different sample rates resulting in two discrete time systems: $H_{1}$ sampled at frequency $f_{s 1}=\frac{1}{T_{e}}$ and $H_{2}$ sampled at frequency $f_{s 2}=\frac{N}{T_{e}}$; and let us compare the truncation error of each one after $T_{e}$ seconds for $N$ large enough. The discrete time is given by $t_{H 1}=n T_{e}$ for $H_{1}$ and $t_{H} 2=k \frac{T_{e}}{N}$ for $H_{2}$, with $n, k \in \mathbb{N}$. Assume that the initial times and the initial conditions are the same for both of them, that is: $n T_{e}=k \frac{T}{T_{e}}=t_{0}$ and $x\left(n T_{e}\right)=x\left(k \frac{T_{e}}{N}\right)=x\left(t_{0}\right)=x_{0}$, The dynamics of the discrete time system $H_{1}$ can be written as: 


$$
x\left((n+1) T_{e}\right)=x\left(n T_{e}\right)+T_{e} f\left(x\left(n T_{e}\right)\right)+O\left(T_{e}^{2}\right)
$$

$H_{2}$, sampled at $f_{s 2}=\frac{N}{T_{e}}$, evolves as follows:

$$
\begin{aligned}
x\left((k+1) \frac{T_{e}}{N}\right)= & x\left(k \frac{T_{e}}{N}\right)+\frac{T_{e}}{N} f\left(x\left(k \frac{T_{e}}{N}\right)\right)+O\left(\frac{T_{e}}{N}\right)^{2} \\
x\left((k+2) \frac{T_{e}}{N}\right)= & x\left((k+1) \frac{T_{e}}{N}\right)+\frac{T_{e}}{N} f\left(x\left((k+1) \frac{T_{e}}{N}\right)\right)+O\left(\frac{T_{e}}{N}\right)^{2} \\
= & x\left(k \frac{T_{e}}{N}\right)+\frac{T_{e}}{N} f\left(x\left(k \frac{T_{e}}{N}\right)\right) \\
& +\frac{T_{e}}{N} f\left(x\left(k \frac{T_{e}}{N}\right)+\frac{T_{e}}{N} f\left(x\left(k \frac{T_{e}}{N}\right)\right)+O\left(\frac{T_{e}}{N}\right)^{2}\right)+2 O\left(\frac{T_{e}}{N}\right)^{2}
\end{aligned}
$$

For $N$ large enough we can consider the influence of the error term $O\left(\frac{T_{e}}{N}\right)^{2}$ over the function $\frac{T_{e}}{N} f(\cdot)$ as a term in $O\left(\frac{T_{e}}{N}\right)^{3}$, then:

$$
\begin{aligned}
x\left((k+2) \frac{T_{e}}{N}\right) \approx & x\left(k \frac{T_{e}}{N}\right)+\frac{T_{e}}{N} f\left(x\left(k \frac{T_{e}}{N}\right)\right)+\frac{T_{e}}{N} f\left\{x\left(k \frac{T_{e}}{N}\right)+\frac{T_{e}}{N} f\left(x\left(k \frac{T_{e}}{N}\right)\right)\right\} \\
& +2 O\left(\frac{T_{e}}{N}\right)^{2} \\
= & x\left(k \frac{T_{e}}{N}\right)+\frac{T_{e}}{N} f\left(x\left(k \frac{T_{e}}{N}\right)\right)+\frac{T_{e}}{N} f\left(x\left((k+1) \frac{T_{e}}{N}\right)\right)+2 O\left(\frac{T_{e}}{N}\right)^{2}
\end{aligned}
$$

So, in a general way, we have:

$$
x\left((k+N) \frac{T_{e}}{N}\right) \approx x\left(k \frac{T_{e}}{N}\right)+\frac{T_{e}}{N} \sum_{i=k}^{k+N-1} f\left(x\left(i \frac{T_{e}}{N}\right)\right)+N O\left(\frac{T_{e}}{N}\right)^{2}
$$

As we can see from (56) and (59), the truncation errors of the discrete systems $H_{1}$ and $H_{2}$ are

$$
\varepsilon_{1}=O\left(T_{e}^{2}\right)
$$

and

$$
\varepsilon_{2}=N O\left(\left(\frac{T_{e}}{N}\right)^{2}\right)
$$

The truncation errors $\varepsilon_{1}$ and $\varepsilon_{2}$, given by (60) and (61) respectively give

$$
N O\left(\left(\frac{T_{e}}{N}\right)^{2}\right) \approx \frac{O\left(T_{e}^{2}\right)}{N}
$$




$$
\varepsilon_{2} \approx \frac{\varepsilon_{1}}{N}
$$

Then the oversampled system $\mathrm{H}_{2}$ reduces the truncation error about $\mathrm{N}$ times.

In practice, to achieve the benefits of oversampling, we emulate this technique based on the assumption that between two consecutive samples of an input signal, its derivative is nearly constant. In this way the new" samples are obtained by linear interpolation between consecutive "measured" samples. This technique is shown in figure 5. On the top the
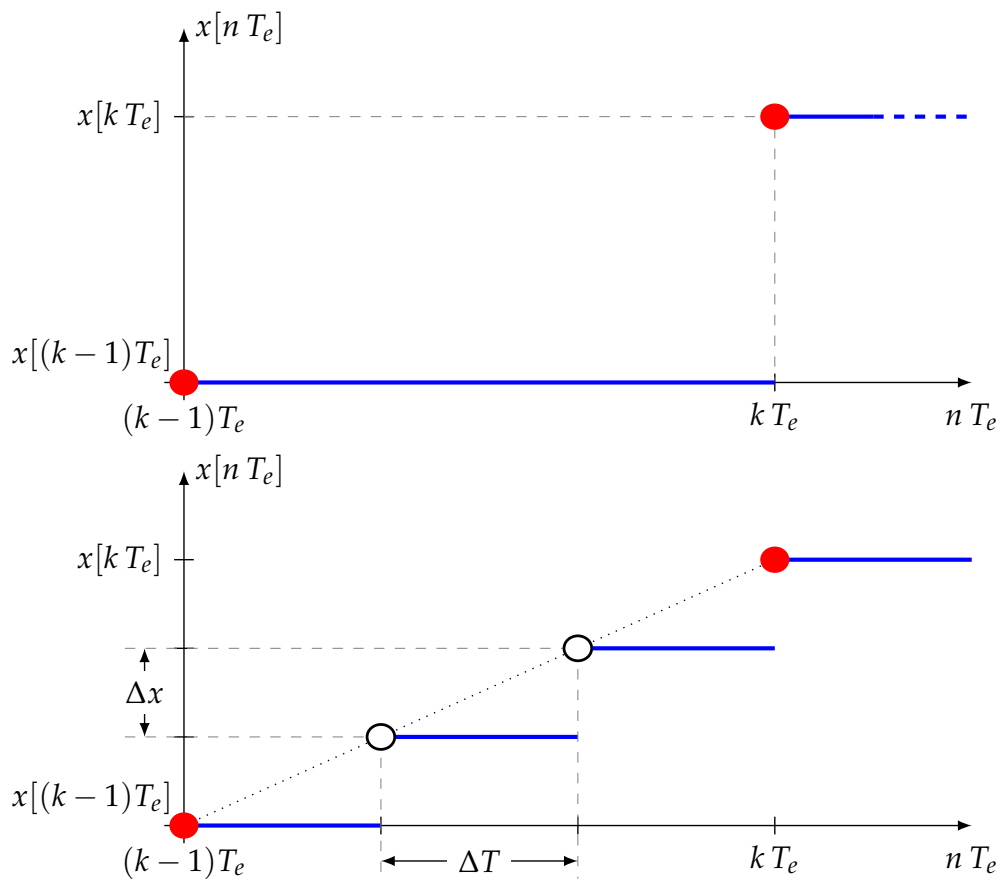

Figure 5. Comparison between sampling and oversampling implementation.

"classical sampling" is shown and on the bottom, the oversampling technique is depicted. As we can see, the sample period is reduced three times its original value, that is, $\frac{T_{e}}{N}=\frac{T_{e}}{3}$. This technique reduces the truncation error, inherent to Euler's method, three times. The benefits of this technique are exposed and validated by experimental tests.

\section{Experimentations}

\subsection{Test bench}

Table (1) presents all electrical and mechanical parameters of Induction machine used in practice, and in Table (2) main VAR-CNTRL card features are presented.

The tunning parameters $\alpha_{i}, \lambda_{i}, i=1, \ldots, 4$ of the proposed observer are chosen according to inequalities (31), 32, 33 and 34 to satisfy convergence conditions. 


\begin{tabular}{|l|l|c|}
\hline$P_{N}$ & rated power & $1.5 \mathrm{KW}$ \\
$V_{N}$ & rated voltage & $230 \mathrm{~V}$ \\
$I_{N}$ & rated current & $3.2 \mathrm{~A}$ \\
$F_{N}$ & rated frequency & $50 \mathrm{~Hz}$ \\
$N_{N}$ & rated speed & $2998 \mathrm{tr} / \mathrm{min}$ \\
$\mathrm{p}$ & number of pair of poles & 1 \\
$R_{S}$ & stator resistance & $4.2 \Omega$ \\
$R_{R}$ & rotor resistance & $2.8 \Omega$ \\
$L_{S}$ & stator inductance & $0.522 \mathrm{H}$ \\
$L_{R}$ & stator inductance & $0.537 \mathrm{H}$ \\
$M_{S R}$ & mutual inductance & $0.502 \mathrm{H}$ \\
$\mathrm{f}$ & viscous coefficient & $1 \mathrm{~N} . \mathrm{s} / \mathrm{rad}$ \\
\hline \hline$\alpha_{1}, \lambda_{1}$ & tunning parameters & $\alpha_{1}=1500, \lambda_{1}=2500$ \\
$\alpha_{2}, \lambda_{2}$ & tunning parameters & $\alpha_{2}=\alpha_{1}, \lambda_{2}=\lambda_{1}$ \\
$\alpha_{3}, \lambda_{3}$ & tunning parameters & $\alpha_{3}=1500, \lambda_{3}=2000$ \\
$\alpha_{4}, \lambda_{4}$ & tunning parameters & $\alpha_{4}=\alpha_{3}, \lambda_{4}=\lambda_{3}$ \\
\hline
\end{tabular}

Table 1. Induction machine and observer parameters.

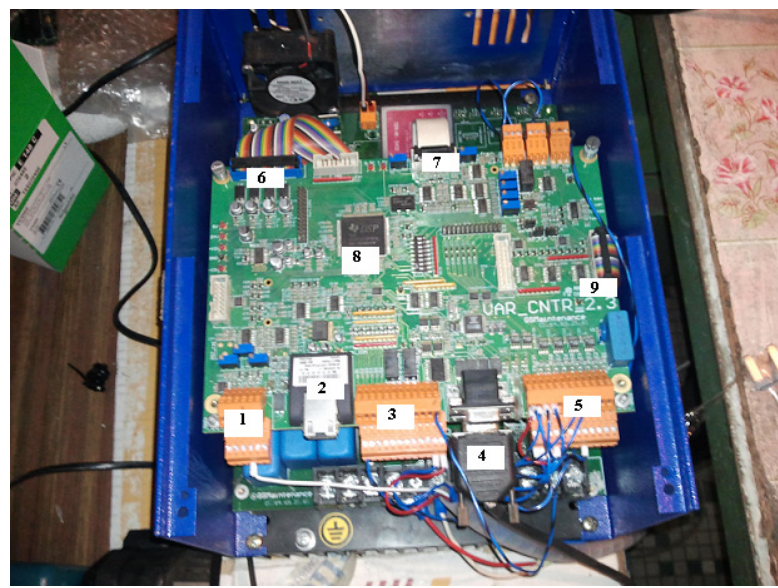

Figure 6. VAR-CNTRL card a product of GS Maintenance.

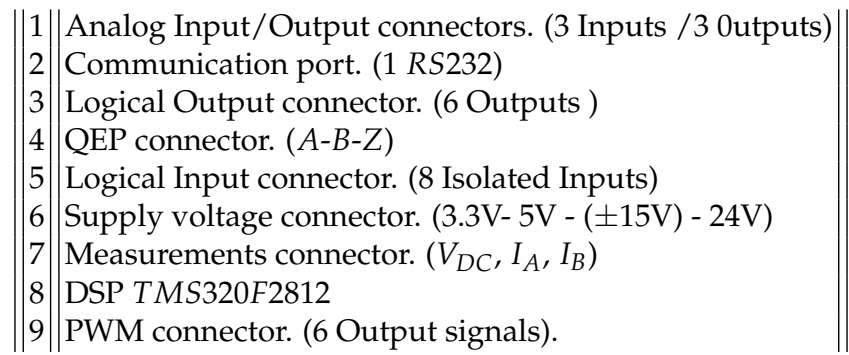

Table 2. VAR-CNTRL card main elements. 
VAR-CNTRL is a electronic card designed by GS Maintenance and dedicated to motor control ( Synchronous, Induction machine, Brushless, and DC motor). Equipped with a DSP TMS320F2812 from Texas Instrument, this component is a fixed point; data are represented under 32 bits.

Practicals tests have been done under the following configurations:

- Fe , Sampling frequency of 8KHZ.

- Fcyc, DSP clock frequency of 150MHZ.

- 1024 points encoder, as speed sensor.

- $\quad$ ADC's (Analog-to-Digital Coder) of 12 bits provide bus voltage $\left(V_{D C}\right)$, and phase currents $\left(I_{A}, I_{B}\right)$ frames under 12 bits.

In addition to the VAR-CNTRL, a MMI (Man Machine Interface) permits to visualize DSP data registers in representation format 8.8 that means possible variations are from [-127.996 to 128].

To summarize our Bed Test description, we have :

- An IM.

- A two-level VSI (Voltage Source Inverter).

- A control card, VAR-CNTRL.

- A MMI.

- A speed sensor, a voltage sensor, and two current sensors.

\subsection{Results}

In this section we propose some experimentation results, that allow the following points:

- Validate Super Twisting Algorithm convergence.

- Evaluate Oversampling method efficiency.

- Evaluate Motor variables estimation.

In section 4.1 we introduced Euler Explicit Sampling Method to discretize a continuous system. Some technical limits about sampling frequency Fe lead us to introduce Oversampling strategy (c.f. section 4.2) . At first glance we propose to validate Super Twisting Observer strategy (c.f. system 7), we will take account of subsystem $\Sigma_{1}$ in figure 4 with the following entries : $\left(v_{1}, z_{1}\right)$, and outputs : $\left(\hat{z}_{1}, \tilde{z}_{3}\right)$.

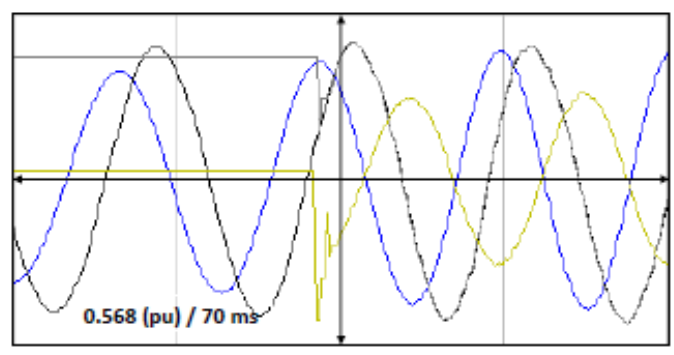

Figure 7. MMI capture : $\left(z_{1}, v_{1}\right)$ and $\left(\hat{z}_{1}, \tilde{z}_{3}\right)$ on convergence phase. 
On figure 7 we validate the convergence of $\Sigma_{1}$ in figure 4 , we can see that under some initials values $\hat{z}_{1}$ converge to $z_{1}$ in a finite time.

Figures 8 and 9 permit to assume that oversampling method is efficient, in fact we see that signals estimated by the observer (53) of the subsystem $\Sigma_{1}$ in figure 4 are much more better with an oversampling than without.

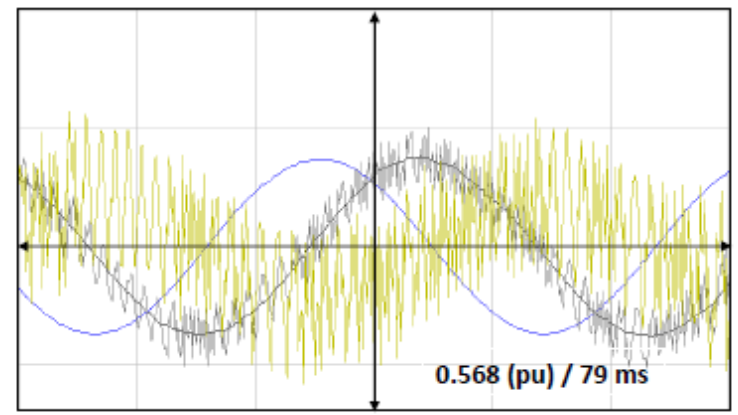

Figure 8. MMI capture : $\left(z_{1}, v_{1}\right)$ and $\left(\hat{z}_{1}, \tilde{z}_{3}\right)$ without oversampling method.

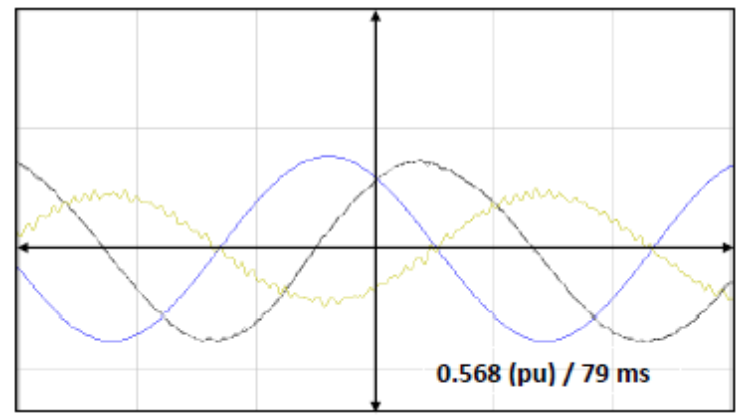

Figure 9. MMI capture : $\left(z_{1}, v_{1}\right)$ and $\left(\hat{z}_{1}, \tilde{z}_{3}\right)$ with oversampling method. $(N=10$.

Thus at the same operating point, we assume that with oversampling method we improve efficiency of the algorithm. With this validated data we can now abort estimation of IM magnetic $\left(x_{3}, x_{4}\right)$ and mechanical $\left(x_{5}\right)$ variables including the rotor position flux $\rho$ given in equations 51, (35-36) and (52) respectively.

The main objective of this work is to provide a motor speed estimation without any mechanical sensor, and then drive it. Note that the speed sensor is only used in comparison of estimated speed with its measure. To validate our strategy we propose some tests into different conditions.

Figures 12 and 13 permit to validate accuracy of estimated speed compare to measured speed in high variation range. However, it is admit that at low and very low speed, estimated speed damages more and more, as we can see on figures 14 and 15.

Now we propose some dynamical test results. During acceleration and deceleration phases (c.f. 16), estimated speed is steel working although there is small delay between $x_{5}$ and $\hat{x}_{5}$. 


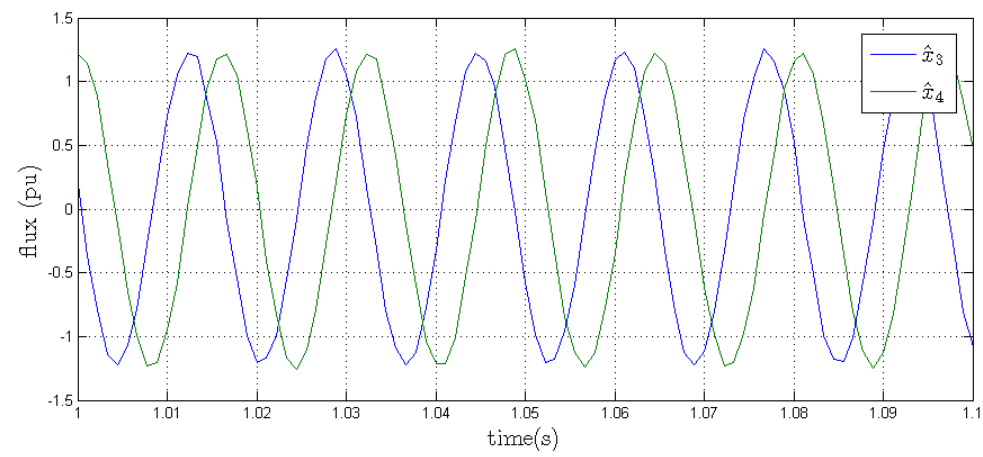

Figure 10. Flux Estimation: $x_{3}$ and $x_{4}$ during static phase.

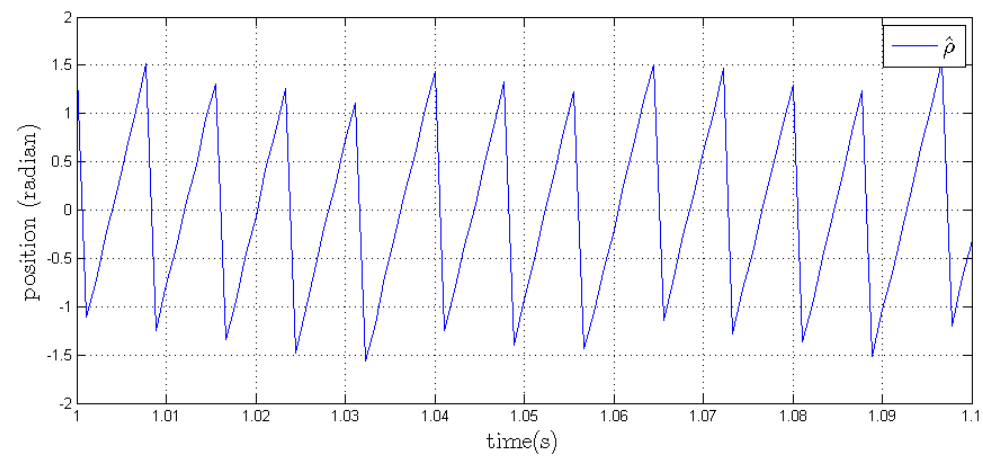

Figure 11. Estimated of rotor flux position: $\rho$.

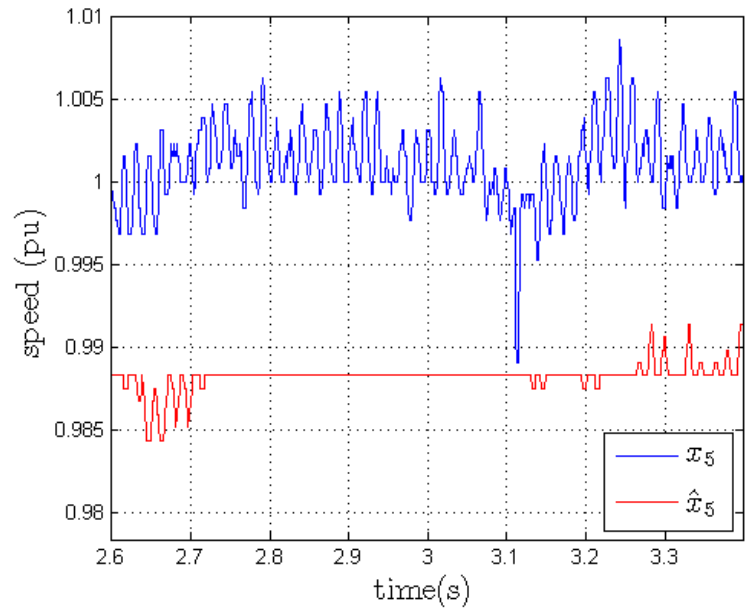

Figure 12. Measure and estimate of speed during static phase: $x_{5}$ and $\hat{x}_{5}$ 


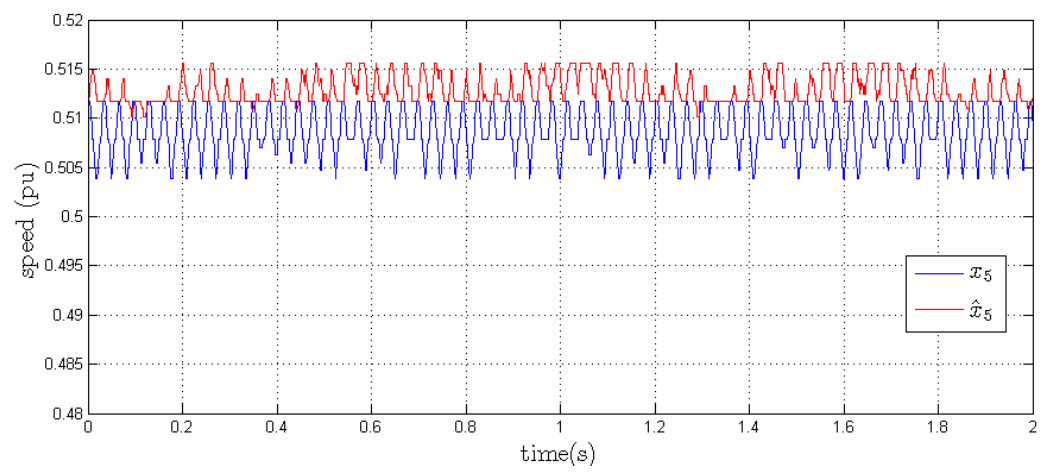

Figure 13. Measure and estimate of speed during static phase: $x_{5}$ and $\hat{x}_{5}$

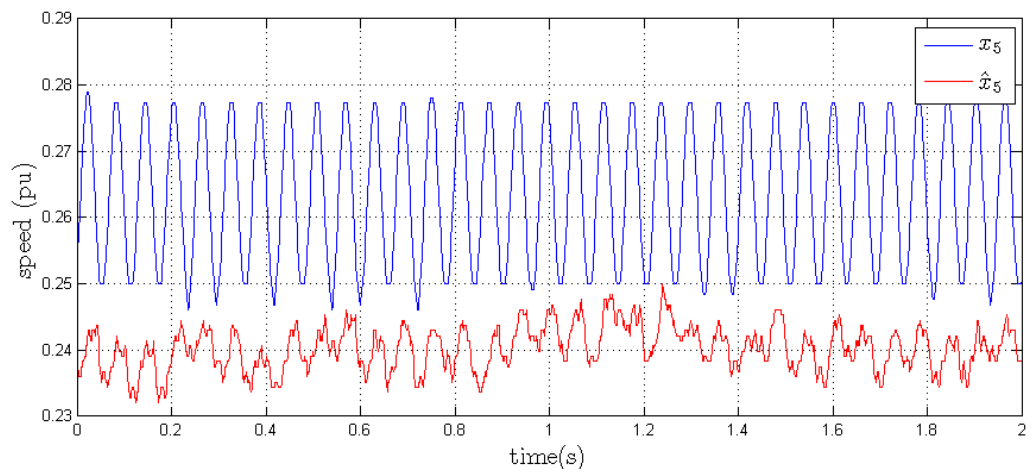

Figure 14. Measure and estimate of speed during static phase: $x_{5}$ and $\hat{x}_{5}$

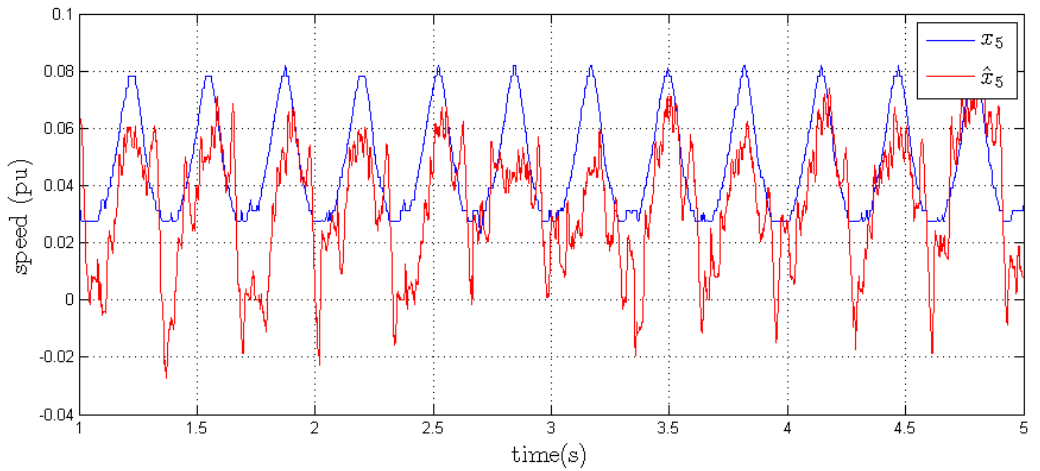

Figure 15. Measure and estimate of speed during static phase: $x_{5}$ and $\hat{x}_{5}$

During static phase operation we saw that at low and very low speed, speed observation does not work very well. However on figures (17) and (18) we cross 0 speed, we denote a small 
divergence as small as the time to cross it; in fact this phenomenon underlines that speed is non observable with low current dynamic.

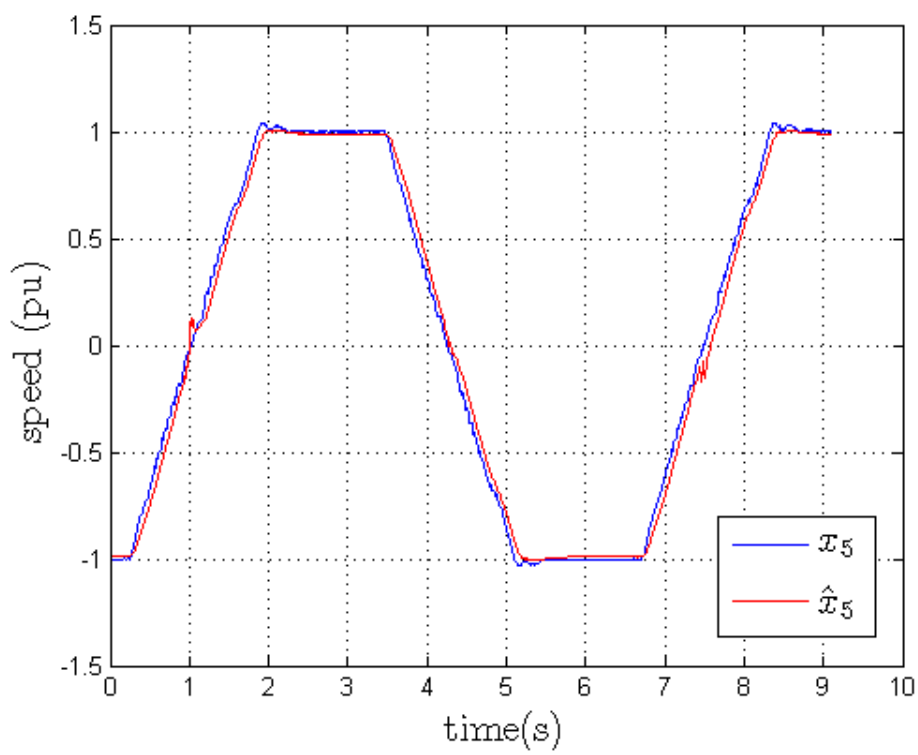

Figure 16. Measure and estimate of speed during variable phase: $x_{5}$ and $\hat{x}_{5}$

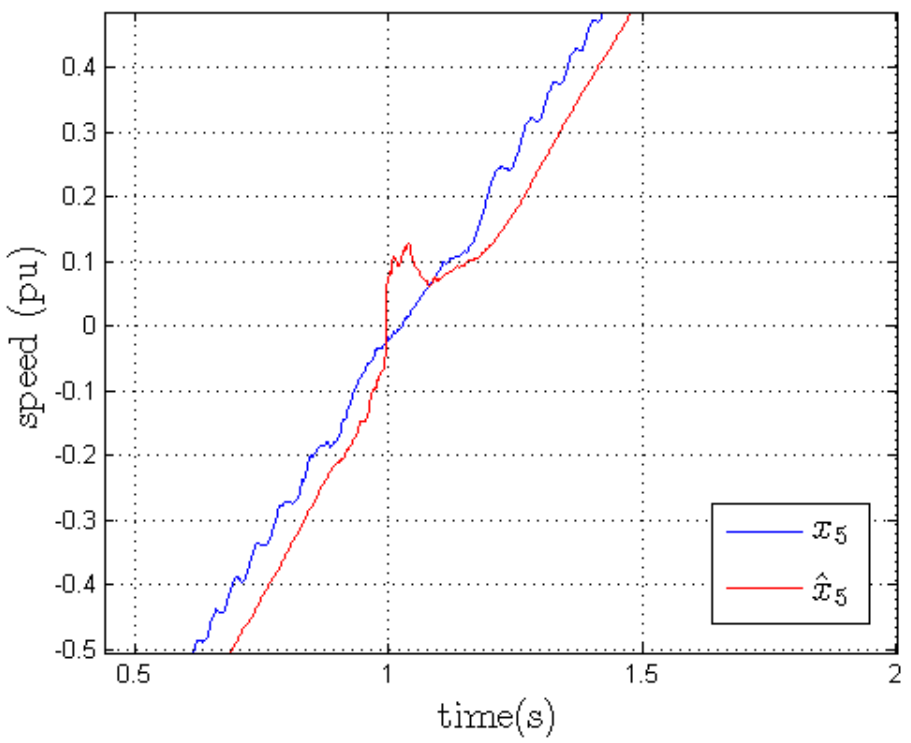

Figure 17. Measure and estimate of speed during acceleration phase: $x_{5}$ and $\hat{x}_{5}$ 


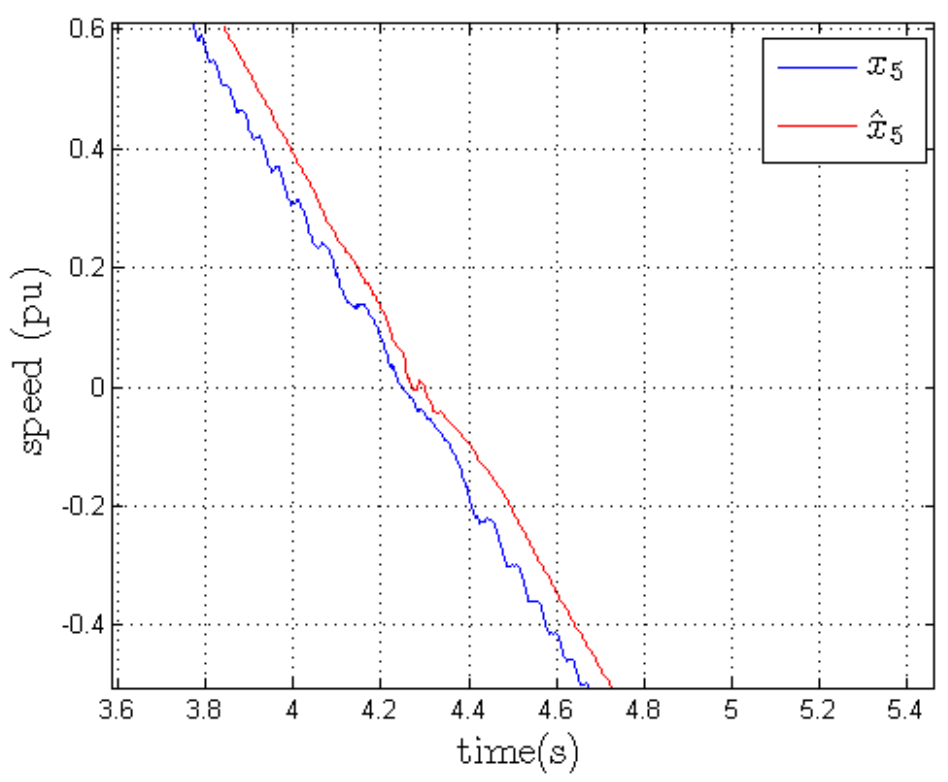

Figure 18. Measure and estimate of speed during deceleration phase: $x_{5}$ and $\hat{x}_{5}$

Figures 12 and 13 at high speed, show that speed approximation proposed in equation (51) work and permit to obtain magnitude and speed sign. This efficiency is also proved during dynamical phases as we can see on figure 16.

About this bad results, we have 2 arguments:

- Parameters error, mainly on stator and rotor resistance $\left(R_{S}, R_{R}\right)$.

- Poor current dynamic, combined to digitizing error at low frequency working.

To overcome all this features, we propose to use an on-line resistor measurement of stator threw temperature.

\section{Conclusion}

Through this chapter an original method of observation without mechanical sensors for induction machine was introduced.

Designed for a embedded system (VAR-CNTRL) equipped with a fixed point DSP, we carried out various tests of validation.

We used concept of Sliding Mode through Super Twisting Algorithm, and oversampling method being based on the explicit Euler development. The contribution of this paper is mainly based on the applicability of the proposed observer for sensorless induction motor when a basic microprocessors are used in an industrial context.

At the time of the setting works of our strategy some technical constraints brought us to introduce a news strategy. 
Thus the practical results permit us to do a first assessment:

- we validate our oversampling method introduced to overcome low speed data acquisition.

- we validate speed estimation during static and dynamic steps.

- we obtained an image of rotor flux $\left(x_{3}\right.$, and $\left.x_{4}\right)$, and also rotor position.

Compared with mechanical sensor the precision provides by the observer on the size speed offer a precision inferior or equal to $5 \%$ in the operating speed range from: $25 \%$ to $100 \%$.

In term of prospects, it possible to improve the threshold of operation in low mode $(25 \%$ to $5 \%$ ) by adaptation oversampling number to stator frequency value, indeed a larger sample number could improve approximation of the continuous system .

In next step some tests will done to validate:

- Validation of hardiness to load variation.

- Validation in closed loop.

About Observability loose at very low speed a first solution could be to switch with a speed estimator.

\section{Author details}

Sebastien Solvar, Malek Ghanes, Leonardo Amet, Jean-Pierre Barbot and Gaëtan Santomenna ECS - Lab, ENSEA and GS Maintenance, France

\section{References}

[1] Aurora, C. \& Ferrara, A. [2007]. A sliding mode observer for sensorless induction motor speed regulation, International Journal of Systems Science 38(11): 913-929.

[2] Bartolini, G., Ferrara, A. \& Usani, E. [1998]. Chattering avoidance by second-order sliding mode control, Automatic control, IEEE Transactions on 43(2): 241-246.

[3] Canudas De Wit, C., Youssef, A., Barbot, J., Martin, P. \& Malrait, F. [2000]. Observability conditions of induction motors at low frequencies, Decision and Control, 2000. Proceedings of the 39th IEEE Conference on, Vol. 3, IEEE, pp. 2044-2049.

[4] Chiasson, J. [2005]. Modeling and high performance control of electric machines, Vol. 24, Wiley-IEEE Press.

[5] Davila, J., Fridman, L. \& Levant, A. [2005]. Second-order sliding-mode observer for mechanical systems, IEEE Transactions on Automatic Control Vol. 50(11): 1785-1789.

[6] Dib, A., Farza, M., MŠSaad, M., Dorléans, P. \& Massieu, J. [2011]. High gain observer for sensorless induction motor, World Congress, Vol. 18, pp. 674-679.

[7] Floquet, T. \& Barbot, J. [2007]. Super twisting algorithm-based step-by-step sliding mode observers for nonlinear systems with unknown inputs, International Journal of Systems Science 38(10): 803-815.

[8] Ghanes, M., Barbot, J., De Leon, J. \& Glumineau, A. [2010]. A robust sensorless output feedback controller of the induction motor drives: new design and experimental validation, International Journal of Control 83(3): 484-497.

[9] Ghanes, M., De Leon, J. \& Glumineau, A. [2006]. Observability study and observer-based interconnected form for sensorless induction motor, Decision and Control, 2006 45th IEEE Conference on, IEEE, pp. 1240-1245. 
[10] Ghanes, M. \& Zheng, G. [2009]. On sensorless induction motor drives: Sliding-mode observer and output feedback controller, Industrial Electronics, IEEE Transactions on 56(9): 3404-3413.

[11] Hilairet, M., Auger, F. \& Berthelot, E. [2009]. Speed and rotor flux estimation of induction machines using a two-stage extended kalman filter, Automatica 45(8): 1819-1827.

[12] Holtz, J. [2006]. Sensorless control of induction machinesƯwith or without signal injection ?, Industrial Electronics, IEEE Transactions on 53(1): 7-30.

[13] Ibarra-Rojas, S., Moreno, J. \& Espinosa-Pérez, G. [2004]. Global observability analysis of sensorless induction motors, Automatica 40(6): 1079-1085.

[14] Khalil, H., Strangas, E. \& Jurkovic, S. [2009]. Speed observer and reduced nonlinear model for sensorless control of induction motors, Control Systems Technology, IEEE Transactions on 17(2): 327-339.

[15] Kubota, H., Matsuse, K. \& Nakano, T. [1993]. Dsp-based speed adaptive flux observer of induction motor, Industry Applications, IEEE Transactions on 29(2): 344-348.

[16] Leppänen, V.-M. [2003]. Low-Frequency Signal-Injection Method for Speed Sensorless Vector Control of Induction Motors, Oxford University Press, ISBN 0-19-856465-1.

[17] Levant, A. [1998]. Robust exact differentiation via sliding mode technique, Automatica 34(3): 379-384.

[18] Levant, A. [2003]. Higher-order sliding modes, differentiation and output-feedback control, International Journal of Control 76(9-10): 924-941.

[19] Maiti, S., Verma, V., Chakraborty, C. \& Hori, Y. [2012]. An adaptive speed sensorless induction motor drive with artificial neural network for stability enhancement, IEEE Transactions on Industrial Informatics .

[20] Perruquetti, W. \& Barbot, J. [2002]. Sliding mode control in engineering, Vol. 11, CRC.

[21] Schauder, C. [1992]. Adaptive speed identification for vector control of induction motors without rotational transducers, Industry applications, IEEE Transactions on 28(5): 1054-1061.

[22] Vas, P. [1998]. Sensorless vector and direct torque control, Vol. 729, Oxford university press Oxford, UK. 